\title{
Reform implementation of research initiatives in a university physics laboratory teaching system
}

\author{
Tao-tao Xu, Bo-lin Xie, Bin Zhao \\ University center of physics teaching laboratory, Wuhan Polytechnic University, Changqing Garden, Wuhan, China
}

\begin{abstract}
Demonstrates the importance of university physics laboratory teaching for the training of college students. Lists some existing issues that affect the development of physics laboratory courses. Reports on efforts to reform research and practical lab-based classes and achievements made from building a new university physics laboratory teaching system.
\end{abstract}

Keywords - university physics laboratory courses, education reform, implementation

\section{大学物理实验教学体系研究和改革的实践}

\author{
徐滔滔 谢柏林 赵斌 \\ 武汉轻工大学大学物理实验教学中心, 常青花园, 武汉, 中国
}

摘 要 认识大学物理实验教学在人才培养中的重要地位, 寻找不利课程发展的问题所在, 研究并实践课程改革的实际方案, 努力 建设实现创新教育的大学物理实验体系。

关键词 大学物理实验, 创新教育, 实践

\section{1. 引言}

大学物理实验是学生进入大学后接触的第一门较为系 统、完整的实验课程。它对学生的基本实验技能、科学实 验素质和创新思维习惯等诸多方面的培养具有不可替代的 作用。然而这门内容广范, 涉及学生众多的课程, 在以往 的实验教学中却存在诸多问题。

(1) 大学物理实验体系设置缺乏系统、层次性。传统 的大学物理实验课程形式比较单一, 基本只对工科学生开 设。无法满足不同层次、不同专业学生的培养需求。课程 素质教育功能难以发挥。建立新的大学物理实验教学体系 势在必行。

(2) 大学物理实验内容陈旧, 功能狭窄。原有的物理 实验一直沿袭传统的教学模式开设实验。多数是验证理论 课堂教学的内容, 实验内容及测量工具陈旧落后。作实验 时学生大多知道实验走向, 没有产生应有的吸引力, 学生 缺乏对实验的热情。这样培养了求同思维, 淡化了求异思 维。不利于学生个性发展, 不利于创新思维的训练。大学 物理实验特别需要引入涉及当今物理科学的最新成就和发
展趋势的相关内容。亟待开发综合性、探究性和设计性实 验。

（3）实验教学方法手段单调, 过多重视实验的结果, 忽视了实验过程。学生进行实验之前, 教师检查实验预习, 讲授实验原理、方法, 甚至示范操作, 然后学生开始实验, 最后学生实验结束检查实验结果。这种灌输式实验教学方 式, 容易造成学生的惰性心理, 不利于培养学生自我获取 信息的意识, 也不利于学生的可持续发展。教学以学生“做 会”实验为基本目标, 只看结果, 不重过程, 忽视了诸如耐 心、自信心和毅力等重要的非智力因素的培养。

（4）实验课程成绩评定缺乏科学性。以往教学对学生 实验课程成绩的评定办法较为单一。不设考核环节, 以平 时实验报告成绩为主。即使有考试也是卷面基本实验知识 的测试, 忽视了学生实验操作过程的具体表现。如此学生 经过一学期、一学年实验课的学习与训练, 就其基本实验 技能提高了多少, 评估缺乏客观依据, 更谈不上考察他们 实验问题的解决能力、综合实验素质, 难以对学生进行科 学的全面评价。

湖北省高等学校省级教学研究项目支持（序号：2012315） 
针对大学物理实验教学的实际, 武汉轻工大学大学物 理实验教学中心教师积极开展研究, 制定改革方案, 努力

创建适应高素质人才培养的大学物理实验教学新体 系。我们的具体做法如下。

\section{2. 新订教学大纲, 构建多层次、多功能大学物理实 验教学体系}

根据《普通高等学校本科教学工作水平评估指标体系》 和教育部颁布的《基础课实验教学示范中心》建设标准, 结合我校实际特点, 我们先后修订和增订了《大学物理实 验 I》(60 学时)、《大学物理实验 II》(30 学时)、《物理现 象释疑》(30 学时)、《“帕斯克”物理探索》(30 学时)、《近 代物理实验》(30 学时) 等课程的教学大纲。将四年本科教 学中各门物理实验课程作为一个整体通盘考虑。以能力和 素质培养为主线, 将各个实验模块进行重组与整合。建立 了由基础到前沿, 由接受式到自主型, 逐步升级的“基础型 一提高型一研究型” 三大层次物理实验教学体系。满足了全 校各专业 (包括文科) 学生的不同培养需求, 更加适合于 现代教育的发展。以力争“扭转目前物理教学中由于“应试教 育'可能给予学生某些被 '题海' 歪曲了的物理世界的图像, 从而实现个人的智力、智慧和创造力的发展与科学知识、 科学体系的形成过程达到基本平行和同步”[1]。

\section{3. 加大设备投入, 改善教学基本条件}

实验体系的建设以设备投入为基础。05 年以来物理实 验中心共投入经费 390 万元, 购买固定资产设备约 610 台 套, 进行了多方面的建设。使物理实验项目达到工科物理 实验教学的基本要求, 装备水平达到教育部规定的物理实 验课程的建设标准。

\section{1 更新、增补基础物理实验台套数, 保证基础物理实验}

随着教育的发展和学校规模的壮大, 更新和增补基础 物理实验是非常必要的。一些如简单验证性的实验, 已经 在生产或科研上不再使用的落后实验手段和仪器必须删 减; 招生人数的增加, 一人一组的基本教学要求必须保证, 这就必然要增补实验设备台套。在淘汰落伍实验项目的同 时, 实验室通过自制仪器和购买设备新开出了 “热敏电阻数 字温度计的设计”、“超声光栅测声速”、“双光栅测弱振动”、 “密立根油滴实验”等综合性和设计性物理实验项目。

\section{2 新建现代测试物理实验室, 开设《“帕斯克”物理探索》 选修课}

美国 PASCO 公司是全球生产教学仪器品牌公司, 其生
产物理实验设备的指导思想是基于力学、热学、声学、光 学、电学、磁学和近代物理的基本原理, 利用物理的多种 测量方法, 经过传感器测试采集数据, 在计算机上进行量 化分析和数据处理, 从而得出实验结论。2005 年我们最初 投入经费 40 万元, 引进 15 项物理实验仪器, 合计 21 台套 设备。完成策划、设计方案, 编印讲义和实施步骤等开发 工作, 建立了 PASCO 物理实验室。开出《“帕斯克”物理探 索》选修课。选修课一经开出受到了学生的普遍欢迎。 PASCO 实验室不只是仪器装备先进, 更重要的是整个实验 过程具有探索性, 实验步骤由学生自主设计, 实验完成后 由学生自行撰写各具特色的实验报告。 PASCO 实验室为有 探索精神和创造潜力的学生提供了良好的学习训练场所。

3.3 建立近代物理实验室, 开设《近代物理实验》选修课

近代物理是大学物理理论重要的部分, 教育部规定的 《基础课实验教学示范中心》建设标准, 对近代物理实验 项目给出了相当的比例。通过共建基金，我们起步投资 40 万元, 购置相关设备仪器 20 台套, 如“测光速实验”、“拉曼 实验”、“单光子实验”等, 新建了近代物理实验室。开设了 《近代物理实验》选修课。增开近代物理实验, 提高了大 学物理实验的层次, 完善了大学物理实验知识体系。

\section{4 建立演示物理实验室, 增设计算机仿真实验项目}

演示实验是物理理论教学的补充和完善, 它使物理内 容更具有多元性、趣味性, 能更好地营造物理教学的氛围。 使得“你的课堂是一个让你和你的学生激动的地方”[2]学生 从实验现象的观察中体验、思考、探索物理本质, 培养学 生研究问题的兴趣和能力。通过共建基金等, 我们投入演 示实验经费 43 万元, 购买演示仪器设备 130 台套, 创建了 物理演示实验室。物理演示实验以随堂演示, 课后演示, 选修课探究三种形式为学生提供服务。《物理现象释疑》选 修课自 06 年开设至今, 受到了文管类学生的欢迎。

另外为了解决一些物理实验的设备仪器昂贵及实验操 作安全条件要求苛刻的问题, 我们还增设了计算机仿真物 理实验项目。这样一方面扩展了学生视野, 另一方面也缓 解了设备资金投入相对不足的状况。目前计算机仿真物理 实验已开设“凯特摆测重力加速度”、“阿贝比长仪测氢原子 光谱”等项内容。

大学物理实验经过近几年的硬件投入, 实验平台初具 规模, 仪器装备水平更上一层楼, 为实践环节的人才培养 创造了良好的条件。 


\section{4. 改革实验考核办法, 促进学生综合实践能力提高}

科学的考核方法和标准不仅能正确地评价学生的能力 和水平, 同时还能对学生的实验课程学习起到很好的引导 和激励作用。我们学校的大学物理实验课程考核经历了: (1)平时考核、(2)理论卷面考核、(3)常规操作考核（做过的 实验内容)、(4)闭卷理论加常规操作考核、(5)闭卷理论加 应用实验操作考核五个阶段。实践表明前 4 种考核方法都 存在多的弊端。如果只有平时考核和期末理论考试, 学生 的操作技能、综合素质体现不出来; 如果只有操作考核, 学生的实验理论水平反映不够全面; 操作考核平时实验做 过的内容, 其负面结果是有些学生为了应付考试, 可能产 生“背实验、背数据”的行为, 不利于真实考察学生实验能 力。因此近七年来我们一直坚持第(5)种方法的考核。理论 闭卷 (占 30\%), 主要考察学生平时实验所获取的基本实验 理论知识。包括实验原理、仪器使用要领、实验方法、数 据处理、操作要点、操作技巧以及部分实验的应用等。操 作开卷（占 70\%)。临考前进行抽签或教师指定操作一题。 客观考核学生经过一学期或一学年物理实验课程学习后具 备的综合实验能力和素质。由于考核操作涉及新的实验内 容, 这就促使学生平时实验更加要独立思考、独立操作、 独立完成。平时考核与期末考核各占课程总成绩的 $50 \%$ 。 实验成绩全部实行计算机管理, 总成绩不及格者, 必须重 修大学物理实验课程。

\section{5. 开展第二课堂活动, 为学生营造有意义的探索经 历}

大学物理实验课程教学的课内学时总是有限的。大学 生要很好地进行实践环节的互动、合作活动, 课内计划教 学远远满足不了同学们探索需求。第二课堂活动开辟了大 学物理实验教学的新阵地, 得到众多老师和广大学生的积 极支持、参与, 在大学物理课程学习和学生自我成长方面 都达到良好的效果。积极开展第二课堂活动, 是为大学生 拓展动脑、动手训练场所的有效途径; 是挖掘同学潜能、 发挥长项及特质的有益平台。在这里学生能趋于“达到“持续 的、近乎完美的表现 " '[3]。大学生第二课堂活动不仅仅是 “丰富学生课外生活”, 还有重要的“育人”功能。大学生第 二课堂活动的特点可归纳为: 第一, 自发灵活性。第二课 堂的任何活动都是学生自愿参加的, 没有第一课堂教学计 划和教学制度规定的那种强制性。第二, 知识广泛性。第 二课堂内容不受课程大纲限制更加广泛, 可以涉猎所有自 然科学、社会科学, 以及实际生活的各个领域。第三, 趣 味丰富性。第二课堂活动安排以学生兴趣为主要考量。学 生向往知道的; 学生有兴趣探索的; 学生急于想尝试的...... 而且活动形式可以灵活多样, 更具有吸引力。针对第二课
堂的特点, 我们认定大学物理实验教学第二课堂的目的为:

(1) 拓宽学生知识面, 提高对物理学习的兴趣。

(2) 培养学生探索精神, 提高学生科学素养。

(3) 培养动手能力和解决问题的能力。

近年来我们在以下方面进行了有益的尝试。

\section{1 开设自主物理实验室}

让物理理论课程成绩达到 80 分以上的学生自愿进入自 主物理实验室。这些同学在实验室内自选二个以上实验项 目。通过查资料, 自行拿出实验方案, 自主完成实验操作, 并写出有特色的实验项目报告。学期内通过定期的公开实 验答辩, 成绩优秀者将获得学期大学物理实验课程免试的 荣誉。一位曾经获得课程免试荣誉的学生, 十分兴奋地感 言: 这种进行实验方式, 虽然我们自己投入的时间、精力 要多得多, 但同时我们的收获也大得多。

\section{2 鼓励申报校级创新实验项目}

对于物理课程爱好的学生, 老师引导他们申报校级创 新实验项目。在老师的指导下, 同学们经历查询资料、讨 论摸索、设计制作、测试整理等曲折过程实施项目。目前 取得二类成果。完成了一批物理实验项目的开发, 例如: “动 态法测量固体材料的杨氏模量”、“利用 PN 结特性测量波尔 兹曼常数”、“微波特性的研究”、“氢燃料电池工作性能研究” 等。完成了一批简易物理演示教具的制作, 例如: “经历碰 撞的险境”、“数字电表的制作”、“小罐自行吸水”等。这样 一方面为物理实验教学充实了新内容, 另一方面眼见通过 自身努力获得的成果, 更进一步点燃了学生再探索再创造 的激情。

\section{3 组织大学生参与学科竞赛活动}

围绕知识的实际应用和解决问题的能力培养开展学科 竞赛活动。通过物理实验竞赛, 激发学生获取未知知识, 转化所学知识, 综合运用知识的动力。组织和开展每年度 的校级大学物理课程竞赛 (已持续六届), 学生们踊跃参赛。 通过理论笔试和实验操作两轮角逐, 每年都有二、三十名 选手分获一、二、三等竞赛大奖, 优胜者遍布学校九个理 工科类专业学院。积极推进和鼓励学生参加“湖北省大学生 物理实验创新设计大赛”, 在 2010 年、2012 年、2014 年已 举行的三届省级物理实验设计竞赛中, 我校学生作品参赛 数量、获奖数目、得奖等级连届创新记录。三届共有 26 项 作品参赛, 其中获得三等奖作品 10 项、二等奖作品 5 项, 一等奖作品 2 项。学校连续三届均被授予省级物理实验大 
赛优秀组织奖的荣誉。

\section{6. 结论}

大学物理实验教学体系改革与建设是一个复合的系统 工程。在教学中心教师共同的努力下, 大学物理实验课程 的教学体系、教学内容、教学模式的改革取得辉煌进展。 多层次物理实验课程的开出与探索、物理实验辅助教学和 管理网络平台的建设、大学生物理实验第二课堂活动的开 展、科学规范的实验课程考核评价体系的设置, 对培养学 生的科学兴趣, 提高学生创新意识和综合素质, 促进人才 培养质量提高等方面产生明显效果。武汉轻工大学大学物 理实验教学中心 2009 年荣获湖北省高等学校教学成果一等 奖一项。以大学物理实验教学中心教师为主体的教学集体
2010 年被评为省级教学团队。含大学物理实验内容的大学 物理课程 2014 年申报省级精品资源共享课程。在我们的继 续努力中, 定会为社会培养创新型应用人才作出更大的贡 献。

\section{参考文献(References)}

[1] H.-X. Zhu, Physical Education and Prospect. Shanghai: East China Normal University Press, 2002.

[2] L. C. Holt, Instructional Patterns. Shanghai: East China Normal University Press, 2008.

[3] M. Buckingham and D. O. Clifton, Now, Discover Your Strengths. Beijing: China Youth Publishing Group, 2007. 